\title{
Use of a Comprehensive Geriatric Assessment to Predict Short-Term Postoperative Outcome in Elderly Patients With Colorectal Cancer
}

\author{
Yoon Hyun Lee, Heung-Kwon Oh, Duck-Woo Kim, Myong Hoon Ihn ${ }^{1}$, Jee Hyun Kim², Il Tae Son, \\ Sung Il Kang, Gwang Il Kim², Soyeon $\mathrm{Ahn}^{3}$, Sung-Bum Kang \\ Department of Surgery, Seoul National University Bundang Hospital, Seongnam; ${ }^{1}$ Department of Surgery, Soonchunhyang University Gumi \\ Hospital, Gumi; ${ }^{2}$ Department of Internal Medicine, Seoul National University Bundang Hospital, Seongnam; ${ }^{3}$ Division of Statistics, Medical \\ Research Collaborating Center, Seoul National University Bundang Hospital, Seongnam, Korea
}

Purpose: This study was conducted to identify the effectiveness of a preoperative comprehensive geriatric assessment (CGA) for predicting postoperative morbidity in elderly patients who underwent surgery for colorectal cancer.

Methods: Elderly patients ( $\geq 70$ years old) who underwent surgery for colorectal cancer at a tertiary hospital in Korea were identified, and their cases were analyzed using data from a prospectively collected database to establish an association between major postsurgical complications and 'high-risk' patient as defined by the CGA.

Results: A total of 240 patients, with a mean age of $76.7 \pm 5.2$ years, were enrolled. Ninety-five patients (39.6\%) were classified as "high-risk" and 99 patients $(41.3 \%)$ as having postoperative complications. The univariate analysis indicated that risk factors for postoperative complications were age, American Society of Anesthesiologists physical status classification, serum hemoglobin, carcinoembryonic antigen, cancer stage, and "high- risk" status. The multivariable analyses indicated that "high-risk" status (odds ratio, 2.107; 95\% confidence interval, 1.168-3.804; $\mathrm{P}=0.013$ ) and elevated preoperative carcinoembryonic antigen (odds ratio, 2.561; 95\% confidence interval, 1.346-4.871, $\mathrm{P}=0.004$ ) were independently associated with postoperative complications. A multivariable analysis of the individual CGA domains indicated that high comorbidities and low activities of daily living were significantly related with postoperative complications.

Conclusion: A preoperative CGA indicating "high-risk" was associated with major postoperative complications in elderly patients who underwent surgery for colorectal cancer. Thus, using the CGA to identify elderly colorectal-cancer patients who should be given more care during postoperative management may be clinically beneficial.

Keywords: Colorectal neoplasms; Comprehensive geriatric assessment; Complication

\section{INTRODUCTION}

Korea and many other developed countries have increasing percentages of elderly people. The Korea National Statistical Office

Received: August 30, 2016 - Accepted: October 7, 2016

Correspondence to: Duck-Woo Kim, M.D.

Department of Surgery, Seoul National University Bundang Hospital, 82,

Gumi-ro 173beon-gil, Bundang-gu, Seongnam 13620, Korea

Tel: +82-31-787-7101, Fax: +82-31-787-4052

E-mail:kdw@snubh.org

(C) 2016 The Korean Society of Coloproctology

This is an open-access article distributed under the terms of the Creative Commons Attribution NonCommercial License (http://creativecommons.org/licenses/by-nc/4.0) which permits unrestricted noncommercial use, distribution, and reproduction in any medium, provided the original work is properly cited. has predicted that the elderly will account for $38.2 \%$ of the Korean population in 2050, even higher than the $37.7 \%$ expected in Japan [1]. As the aging population increases, the surgery rate for elderly patients is also increasing, which is made possible by improvements in anesthetic and surgical techniques. In the United States, more than half of all operations are performed on elderly patients ( $>65$ years old), and this proportion is increasing [2]. In Korea, the number of individuals older than 60 years of age who were eligible for surgery has increased from 0.47 million in 2004 to 1.05 million in 2014 [1].

Furthermore, elderly patients who underwent head and neck, thoracic, and hip fracture surgery had higher rates of postoperative complications than the general population [3-5]. This may be explained by the greater frailty of the elderly, who often have de- 
creased physiological reserves in multiple organ systems and numerous comorbid conditions. Frail elderly patients are more likely to suffer from postoperative complications such as cardiac problems, pneumonia, and deep vein thrombosis. In spite of the high rate of postoperative complications in the elderly, no optimal tool that accurately predicts postoperative morbidity is available to assess preoperative risks and physiological reserves of the elderly.

Geriatricians initially designed the comprehensive geriatric assessment (CGA) as a multidimensional tool to examine the general health status of the elderly based on validated geriatric scales and tests that examine diverse health problems. The results of the CGA can allow development of individualized geriatric intervention programs, and in many sub-fields of geriatrics, the CGA is used to evaluate geriatric conditions that are associated with frailty [6]. The CGAs used in previous studies assessed the functional, physiological, mental, and socioeconomic features of elderly patients [6-8]. However, the efficacy of the CGA as a preoperative assessment tool has not been validated for surgery patients. Some previous studies used the CGA to study patients with heterogeneous conditions, including those who underwent orthopedic, neurological, cardiac, or abdominal surgery $[7,8]$.

Colorectal cancer (CRC) is a major cause of cancer-related deaths in Korea, and its incidence has been increasing over the past 25 years [9]. The incidence of CRC reached a peak in the 1970s, and CRC is now the most common malignancy in elderly females and the third-most common malignancy in males. Thus, the number of elderly patients undergoing surgery for CRC has seen a steep increase [9]. However, the usefulness of the CGA in predicting the outcome in elderly patients with CRC has not yet been evaluated. Thus, the purpose of the present study was to identify the efficacy of a preoperative CGA for predicting postoperative morbidity in elderly patients who underwent surgery for CRC.

\section{METHODS}

\section{Patient selection}

A retrospective review of a prospective, Institutional Review Board-approved database was performed to identify elderly patients ( $\geq 70$ years old) who had undergone major elective surgery for primary CRC in the Seoul National University Bundang Hospital from 1 March 2009 to 28 February 2014. All patients who had undergone elective CRC surgery and had preoperative CGA results were enrolled. Patients were excluded if they had taken a CGA survey after surgery or more than 6 months prior to surgery, if they had undergone emergency surgery, or if their CGA results were incomplete. The baseline characteristics of patients included epidemiologic data, medical history, and perioperative and laboratory characteristics such as white blood cell count, hemoglobin, platelet count, prothrombin time, albumin, glucose, aspartate transaminase and alanine transaminase, creatinine, and pre/postoperative carcinoembryonic antigen (CEA).

\section{CGA protocol}

A preoperative CGA was used to assess the condition of each patient's health within 6 months of surgery. All patients were interviewed face-to-face by the geriatric team, which consisted of geriatricians, nurse specialists, dieticians, and pharmacists. The preoperative CGA had 8 domains: burden of comorbidity (Charlson Comorbidity Index, CCI), poly-pharmacy, physical function according to activities of daily living (ADL), instrumental ADL (IADL), cognitive status (Mini-Mental State Examination), risk of postoperative delirium (Nursing Delirium Screening Scale), Korean Geriatric Depression Scale, and nutritional status (mini nutritional assessment). The definition of "deficit" in each domain was the same as in our previous studies [7, 8]: a score of 3 or more for comorbidities [10], the regular use of 8 or more drugs [11], poor physical function assessed by using the $\mathrm{ADL}$, poor physical function assessed by using the IADL [12], cognitive dysfunction assessed by using the Korean version of the Mini-Mental State Examination, severe depression, and malnutrition.

We defined a "high-risk" patient as one who had deficits in two or more domains. A surgical complication with Clavien-Dindo grade II or higher within 30 days of surgery was classified as a major complication [13], and a grade I complication as uneventful.

\section{Ethical approval}

The Institutional Review Board of Seoul National University Bundang Hospital approved this study prior to commencement of data collection and analysis (approval number: B-1504-294-103) and waived the informed consent requirement because it was a retrospective review.

\section{Statistical analysis}

The associations between CGA domains, "high-risk" status, demographic data, laboratory findings, and postoperative major complications were analyzed by using chi-square tests. The associations between explanatory variables and postoperative major complications were determined by using multiple logistic regression analyses with backward elimination. Univariate and multivariate analyses were also used to assess the relationships between each CGA domain and postoperative major complications. A Pvalue less than 0.05 was considered statistically significant. All statistical analyses were performed using IBM SPSS ver. 18.0 (IBM Co., Armonk, NY, USA).

\section{RESULTS}

\section{Patient characteristics}

A total of 324 elderly patients underwent surgery for CRC during the study period. Eighty-four patients were excluded because they received emergency operations $(n=4)$ or did not receive a preoperative CGA $(n=80)$, so 240 patients were ultimately enrolled (Table 1). The mean age was 76.7 years (range, 70-96 years), and 6 patients were older than 90 years of age. Sixty percent of the pa- 
Table 1. Baseline demographic, clinical, and laboratory characteristics of elderly patients who underwent surgery for colorectal cancer $(\mathrm{n}=240)$

\begin{tabular}{lc}
\hline Characteristic & Value \\
\hline Sex & \\
Male & $138(57.5)$ \\
Female & $102(42.5)$ \\
Age (yr) & \\
$70-79$ & $180(75.0)$ \\
$80-89$ & $54(22.5)$ \\
$\geq 90$ & $6(2.5)$ \\
Mean \pm SD & $76.7 \pm 5.20$
\end{tabular}

Body mass index $\left(\mathrm{kg} / \mathrm{m}^{2}\right)$

$\begin{array}{lc}\leq 25 & 161(67.0) \\ >25 & 79(33.0) \\ \text { Mean } \pm \text { SD } & 23.8 \pm 3.67\end{array}$

Hypertension

$\begin{array}{lr}\text { No } & 98(40.8) \\ \text { Yes } & 142(59.2)\end{array}$

\section{Diabetes}

$\begin{array}{lr}\text { No } & 190(79.2) \\ \text { Yes } & 50(20.8)\end{array}$

ASA physical status classification

$\begin{array}{lr}\text { I } & 39(16.3) \\ \| & 174(72.5) \\ \geq|I| & 27(11.2)\end{array}$

Prior operation

$\begin{array}{lr}\text { No } & 99(41.2) \\ \text { Yes } & 141(58.8)\end{array}$

Tumor location

$\begin{array}{lr}\text { Colon } & 150(62.5) \\ \text { Rectum } & 90(37.5)\end{array}$

Pathologic stage

$\begin{array}{lr}0-1 & 53(22.1) \\ 2 & 52(21.7) \\ 3 & 110(45.8) \\ 4 & 25(10.4)\end{array}$

Surgical approach

$\begin{array}{lr}\text { Open } & 80(33.3) \\ \text { Laparoscopic } & 160(66.7)\end{array}$

Co-operation

\begin{tabular}{lr} 
No & $186(77.5)$ \\
Yes & $54(22.5)$ \\
\hline
\end{tabular}

(Continued to the next)
Table 1. Continued

\begin{tabular}{lc}
\hline Characteristic & Value \\
\hline White blood cell $\left(\times 10^{3} / \mathrm{mm}^{3}\right)$ & \\
$\leq 4$ & $212(8.3)$ \\
$>4$ & $28(11.7)$
\end{tabular}

Hemoglobin $(\mathrm{g} / \mathrm{dL})$

$\begin{array}{ll}\geq 12.0 & 122(50.8) \\ <12.0 & 118(49.2)\end{array}$

\section{Platelets $(/ \mu \mathrm{L})$}

$\geq 130,000$

$<130,000$

PT INR

$\begin{array}{cc}\leq 1.2 & 231(96.3) \\ >1.2 & 9(3.7)\end{array}$

Serum Albumin ( $\mathrm{g} / \mathrm{dL})$

$\begin{array}{cc}\geq 3.3 & 229(95.4) \\ <3.3 & 11(4.6)\end{array}$

Glucose (mg/dL)

$\begin{array}{rr}\leq 110 & 147(61.3) \\ >110 & 90(38.7)\end{array}$

AST (U/L)

$\begin{array}{cc}\leq 40 & 227(94.6) \\ >40 & 13(5.4)\end{array}$

ALT (U/L)

$\begin{array}{cc}\leq 40 & 232(96.7) \\ >40 & 8(3.3)\end{array}$

Serum creatinine $(\mathrm{mg} / \mathrm{dL})$

$\leq 1.2$

$>1.2$

Preoperative CEA (ng/mL)

$\leq 5 \quad 159(66.3)$

$>5 \quad 60(33.7)$

Postoperative CEA (ng/mL)

$\leq 5$

$>5$

24 (13.7)

Values are presented as number (\%) unless otherwise indicated.

SD, standard deviation; ASA, American Society of Anesthesiologists; PT, prothrombin time; INR, international normalized ratio; AST, aspartate aminotransferase; ALT, alanine aminotransferase; CEA, carcinoembryonic antigen.

tients had hypertension, and $20 \%$ had diabetes mellitus; moreover, most patients had American Society of Anesthesiologists (ASA) physical status classification of I (healthy) or II (mild systemic disease). Two-thirds of the patients had tumors in the colon, and most patients underwent laparoscopic surgery (66.7\%). Laboratory findings indicated that about half of the patients had 
preoperative anemia; 10 of those patients were given transfusions before surgery. The serum albumin level, reported by several studies as an independent predictor for morbidity [14-16], was normal in most patients. About one-quarter of the patients had elevated levels of serum creatinine, but only one had chronic renal insufficiency and needed dialysis. One-third of the patients had elevated levels of preoperative CEA.

\section{CGA results}

Overall, 95 of the enrolled 240 patients (39.6\%) had deficits in 2 or more domains on the preoperative CGA and were categorized as "high-risk" patients (Table 2). Analysis of the individual domains indicated that nearly half of the patients had a score of 3 or more for comorbidities (diabetes mellitus, cerebrovascular disease, chronic pulmonary disease, etc.), about $10 \%$ were dependent in terms of physical activity measured by using the ADL or the IADL, $42.5 \%$ had mild or severe decreases in cognitive function, and a small number had depression (9.2\%) and delirium (3.8\%).

\section{Postoperative complications}

Of the 240 enrolled patients, 183 patients (76.3\%) experienced postoperative morbidities, and 99 (41.3\%) had major complications (grades II-V) that required medical or surgical intervention (Table 3 ). Two patients $(0.8 \%)$ died, one from postoperative pneumonia and the other from multiorgan failure following abdominal sepsis. The most common complication was mild electrolyte imbalance, but that spontaneously resolved in all patients without specific treatment. Among the major complications, voiding difficulty $(10 \%)$ was the most common. This complication was resolved by using urinary catheter reinsertion for more than 3 days to rest the bladder (17 patients) and administration of an alaadrenergic receptor antagonist (24 patients).

\section{Univariate and multivariate analysis of factors associated with major postoperative complications}

Univariate analyses indicated that advanced age, higher ASA physical status classification, more advanced CRC stage, presence of anemia, elevated serum CEA level, and "high-risk" based on the CGA were significantly associated with major complications (Table 4). These 6 significant variables were analyzed by using multiple logistic regression analyses with backward elimination, in which the factor that had the smallest correlation to postoperative major complications was removed in each stage (Table 5). The results indicated that "high-risk" based on the CGA (odds ratio [OR], 2.107; 95\% confidence interval [CI], 1.168-3.804; P = 0.013 ) and a preoperative CEA level of more than $5 \mathrm{ng} / \mathrm{mL}$ (OR, 2.561; 95\% CI, 1.346-4.871; P = 0.004) were significantly and independently associated with postoperative complications.

We also performed univariate and multivariate analyses to evaluate the effect of each deficient domain on the CGA for grades II-V complications (Table 6). The results indicate that a score of 3 or more for comorbidities (OR, 2.237; 95\% CI, 1.183-4.230; $\mathrm{P}=$
Table 2. CGA ${ }^{\mathrm{a}}$ in eight domains and identification of "high-risk" patients $^{\mathrm{b}}$

\begin{tabular}{|c|c|}
\hline Variable & Value \\
\hline \multicolumn{2}{|l|}{ Comorbidities } \\
\hline $0-2$ & $132(55.0)$ \\
\hline$\geq 3$ & $108(45.0)$ \\
\hline Mean \pm SD & $2.76 \pm 1.14$ \\
\hline \multicolumn{2}{|l|}{ Medication } \\
\hline $0-7$ & 207 (86.3) \\
\hline$\geq 8$ & 33 (13.8) \\
\hline Mean \pm SD & $4.04 \pm 3.59$ \\
\hline \multicolumn{2}{|l|}{ ADL } \\
\hline Dependent & $18(7.5)$ \\
\hline Independent & $222(92.5)$ \\
\hline \multicolumn{2}{|l|}{ IADL } \\
\hline Dependent & $26(10.8)$ \\
\hline Independent & 214 (89.2) \\
\hline \multicolumn{2}{|l|}{ MMSE } \\
\hline $0-24$ & 102 (42.5) \\
\hline$\geq 25$ & $132(57.5)$ \\
\hline Mean \pm SD & $23.5 \pm 5.01$ \\
\hline \multicolumn{2}{|l|}{ SGDS } \\
\hline $0-9$ & 210 (87.5) \\
\hline$\geq 10$ & $22(9.2)$ \\
\hline Mean \pm SD & $3.65 \pm 3.50$ \\
\hline \multicolumn{2}{|l|}{ Delirium } \\
\hline $0-1$ & 205 (85.4) \\
\hline$\geq 2$ & $9(3.8)$ \\
\hline Mean \pm SD & $0.24 \pm 0.56$ \\
\hline \multicolumn{2}{|l|}{ MNA } \\
\hline$<17$ & $15(6.3)$ \\
\hline $17-24$ & $83(34.6)$ \\
\hline$>24$ & 141 (58.6) \\
\hline Mean \pm SD & $23.8 \pm 4.38$ \\
\hline \multicolumn{2}{|l|}{ High risk } \\
\hline No & $145(60.4)$ \\
\hline Yes & 95 (39.6) \\
\hline
\end{tabular}

Values are presented as mean \pm standard deviation (range) or number (\%). CGA, comprehensive geriatric assessment; $A D L$, physical function according to activities of daily living; IADL, instrumental ADL; MMSE, Mini-Mental State Examination; SGDS, Korean Geriatric Depression Scale; MNA, mini nutritional assessment; SD, standard deviation.

"CGA indicates the "deficit" group in each domain. "High-risk patients are those that had deficits in two or more domains.

0.013) and a high ADL score (OR, 16.369; 95\% CI, 1.233-217.2; P $=0.034$ ) were significantly and independently associated with a 
Table 3. Grades of postoperative complications $\mathrm{s}^{\mathrm{a}}$ in elderly patients who underwent surgery for colorectal cancer

\begin{tabular}{|c|c|c|c|c|c|c|}
\hline \multirow{2}{*}{ Complication } & \multicolumn{5}{|c|}{ Grade } & \multirow{2}{*}{ Total } \\
\hline & 1 & II & III & IV & V & \\
\hline Electrolyte imbalance & 36 & - & - & - & - & 36 \\
\hline Self-voiding difficulty & - & 24 & - & - & - & 24 \\
\hline Unknown fever & 15 & 4 & - & - & - & 19 \\
\hline Wound problem & 8 & - & 6 & - & - & 14 \\
\hline lleus & 3 & 7 & 1 & 1 & - & 12 \\
\hline Atelectasis & 10 & 1 & - & - & - & 11 \\
\hline Transfusion & - & 9 & - & - & - & 9 \\
\hline Pneumonia & - & 5 & - & 2 & 1 & 8 \\
\hline Cardiac problem & - & 5 & 1 & 2 & - & 8 \\
\hline Nausea \& vomiting & 7 & - & - & - & - & 7 \\
\hline Delirium & - & 7 & - & - & - & 7 \\
\hline Bleeding & 2 & 3 & - & - & - & 5 \\
\hline DVT \& PTE & 1 & 3 & - & - & - & 4 \\
\hline Fluid collection & - & - & 4 & - & - & 4 \\
\hline Anastomotic leakage & - & - & - & 2 & 1 & 3 \\
\hline UTI & - & 3 & - & - & - & 3 \\
\hline URI & 1 & 2 & - & - & - & 3 \\
\hline Acute cholecystitis & - & - & - & 1 & - & 1 \\
\hline Others & 1 & 3 & 1 & - & - & 5 \\
\hline Total, n (\%) & $84(35.0)$ & $76(31.7)$ & $13(5.4)$ & 8 (3.3) & $2(0.8)$ & $183(76.3)$ \\
\hline
\end{tabular}

Gr, grade; DVT, deep vein thrombosis; PTE, pulmonary thromboembolism; UTI, urinary tract infection; URI, upper respiratory infection.

aclavien-Dindo classification of surgical complications [13].

risk of major complications.

\section{DISCUSSION}

The main finding of the present study is that the results of a preoperative CGA can predict postoperative complications in elderly patients who undergo surgery for CRC. Additionally, among the eight domains in the CGA, the comorbidities and the ADL domains were significantly and independently associated with major postoperative complications. Previous studies reported that advanced age, advanced tumor stage, emergency surgery, blood transfusion, malnutrition, diabetes, and pre-existing cardiac pathology were risk factors for postoperative complications in elderly patients with CRC [17-19].

Traditional health scoring systems are widely used to evaluate elderly patients before major abdominal surgery. These systems include the ASA physical status classification, the acute physiology and chronic health evaluation (APACHE II), estimates of physiologic ability and surgical stress, and the physiological and operative severity score for the enumeration of mortality and morbidity. As the CGA has been adopted in various medical fields, some studies have reported on the use of the CGA as a pre- operative assessment $[7,8]$. As far as we know, the present study is the largest study of patients who underwent elective surgery for CRC and were given preoperative CGAs.

Our results indicate that an elevated preoperative level of serum CEA was significantly associated with postoperative complications. However, in no previous studies was any relationship between preoperative CEA and postoperative complications in elderly patients reported or explained. Because tumor burden may have an indirect effect on morbidity, patients with large tumors usually have elevated CEA levels; furthermore, difficulty during surgery due to the large tumor size may lead to more frequent postoperative complications [20]. Thus, elevated CEA level may be associated with more frequent postoperative complications.

Our results indicated that advanced age and ASA physical status classification were not associated with postoperative complications. Previous studies reported that biological age was a more significant prognostic factor than chronological age in elderly patients $[21,22]$. We found that 5 of the 6 patients who were at least 90 years of age recovered without severe postoperative complications; one 90-year-old male, classified as "high risk" based on the CGA results, had pneumonia and died in spite of aggressive medical treatment and intensive care unit care. This finding supports 


\section{$\begin{array}{rlr}\text { Annals of } & \text { Patients With Colorectic } \\ \text { Coloproctology } & \text { Yoon Hyun Lee, et al. }\end{array}$}

Table 4. Univariate analysis of factors associated with major postoperative complications in elderly patients who underwent surgery for colorectal cancer

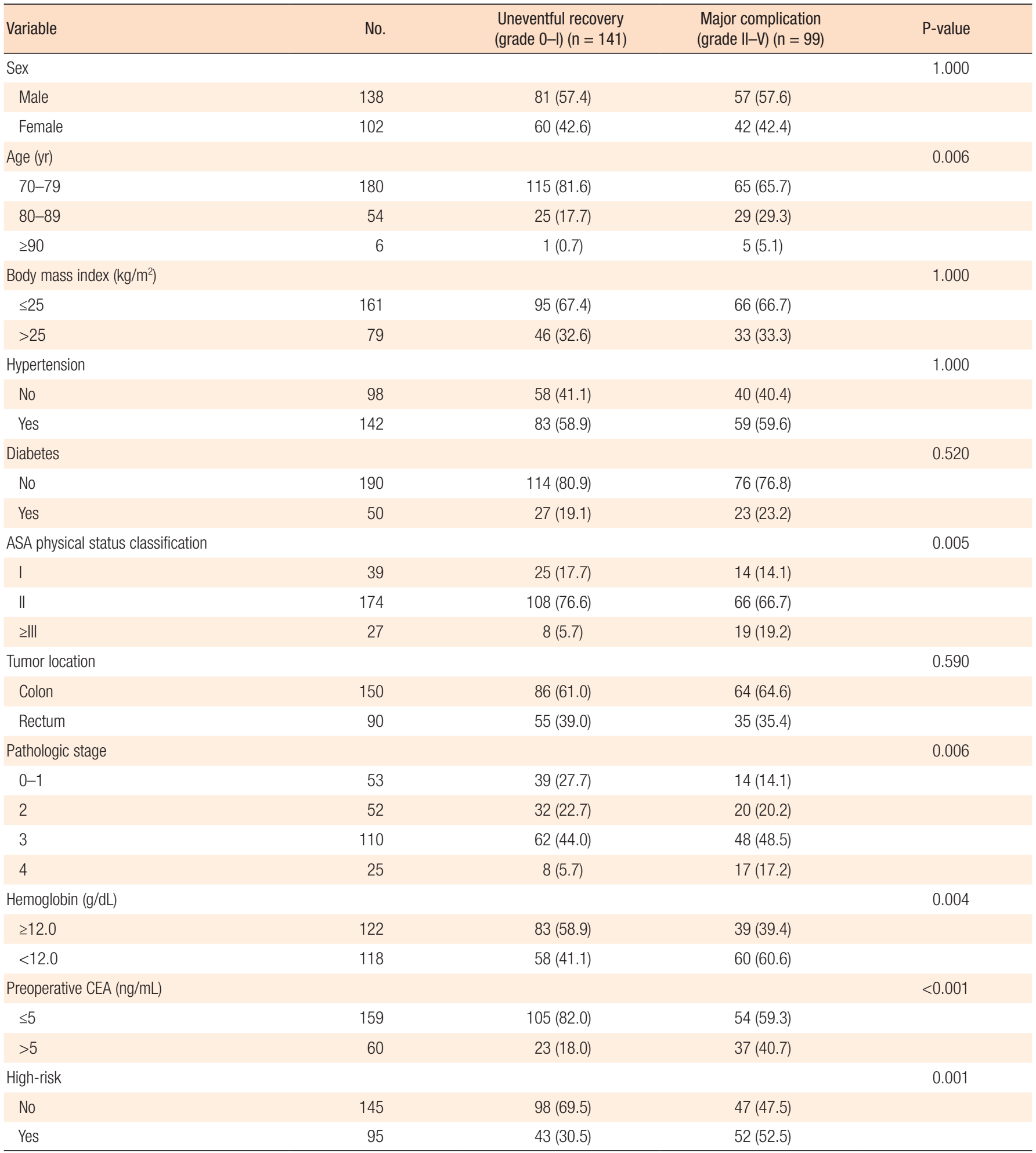

Values are presented as number (\%).

ASA, American Society of Anesthesiologists; CEA, carcinoembryonic antigen. 
Table 5. Multivariate analysis of factors associated with major postoperative complications in elderly patients who underwent surgery for colorectal cancer

\begin{tabular}{llcc}
\hline Characteristic & OR & $95 \% \mathrm{Cl}$ & P-value \\
\hline $\begin{array}{l}\text { No. of deficient domains in CGA } \\
\quad\end{array}$ & 1 & Reference & \\
$0-1$ (low risk) & 2.107 & $1.168-3.804$ & \\
$\geq 2$ (high risk) & & & 0.004 \\
Preoperative CEA (ng/mL) & 1 & Reference & \\
$\leq 5$ & 2.561 & $1.346-4.871$ & \\
$>5$ & &
\end{tabular}

$\mathrm{OR}$, odds ratio; $\mathrm{Cl}$, confidence interval; $\mathrm{CEA}$, carcinoembryonic antigen. the selection of "high risk" patients based on CGA rather than chronological age when planning surgery for elderly patients. The ASA physical status classification is also useful in evaluating elderly surgery patients $[23,24]$. Some recent studies suggested there should be another method rather than ASA physical status classification $[21,25,26]$. The ASA physical status classification was not predictive of morbidity in the present study. The reasons may be the facts that approximately $90 \%$ of our patients were classified as ASA physical status classification I (healthy) or II (mild systemic disease) and the ASA physical status classification may not have the sensitivity to detect differences between those 2 groups.

Table 6. Univariate and multivariate analyses of the relationship of deficit in each CGA domain and occurrence of major postoperative complications

\begin{tabular}{|c|c|c|c|c|c|c|c|}
\hline Characteristic & No. & $\begin{array}{l}\text { Uneventful recovery } \\
\text { (grade } 0-l) \\
(n=141)\end{array}$ & $\begin{array}{l}\text { Major complication } \\
\text { (grade II-V) } \\
(\mathrm{n}=99)\end{array}$ & P-value & $\mathrm{OR}$ & $95 \% \mathrm{Cl}$ & P-value \\
\hline Co-morbidity & & & & 0.004 & & & 0.013 \\
\hline $0-2$ & 127 & $86(61.0)$ & $41(41.4)$ & & 1 & Reference & \\
\hline$\geq 3$ & 113 & $55(39.0)$ & 58 (58.6) & & 2.237 & $1.183-4.230$ & \\
\hline Medications & & & & 0.056 & & & 0.962 \\
\hline $0-7$ & 207 & $127(90.1)$ & 80 (80.8) & & 1 & Reference & \\
\hline$\geq 8$ & 33 & $14(9.9)$ & 19 (19.2) & & 1.023 & $0.392-2.673$ & \\
\hline ADL & & & & $<0.001$ & & & 0.034 \\
\hline Dependent & 28 & $6(4.3)$ & 22 (22.2) & & 16.369 & $1.233-217.2$ & \\
\hline Independent & 212 & $135(95.7)$ & 77 (77.8) & & 1 & Reference & \\
\hline IADL & & & & 0.01 & & & 0.807 \\
\hline Dependent & 26 & $9(6.4)$ & 17 (17.2) & & 0.721 & $0.052-9.915$ & \\
\hline Independent & 214 & $132(93.6)$ & 82 (82.8) & & 1 & Reference & \\
\hline MMSE & & & & 0.18 & & & 0.945 \\
\hline $0-24$ & 102 & $56(40.0)$ & $46(48.9)$ & & 0.977 & $0.509-1.876$ & \\
\hline$\geq 25$ & 132 & $84(60.0)$ & 48 (51.1) & & 1 & Reference & \\
\hline SGDS & & & & 0.36 & & & 0.620 \\
\hline $0-9$ & 210 & $129(92.1)$ & $81(88.0)$ & & 1 & Reference & \\
\hline$\geq 10$ & 22 & $11(7.9)$ & $11(12.0)$ & & 1.302 & $0.459-3.695$ & \\
\hline Delirium & & & & $<0.001$ & & & - \\
\hline $0-1$ & 205 & $129(100)$ & $76(89.4)$ & & 1 & Reference & \\
\hline$\geq 2$ & 9 & $0(0.0)$ & $9(10.6)$ & & - & - & \\
\hline MNA & & & & 0.08 & & & 0.183 \\
\hline$<17$ & 15 & $7(5.0)$ & $8(8.1)$ & & 0.105 & $0.007-1.642$ & \\
\hline $17-24$ & 83 & $42(30.0)$ & $41(41.4)$ & & 1.281 & $0.657-2.500$ & \\
\hline$>24$ & 141 & $91(65.0)$ & $50(50.5)$ & & 1 & Reference & \\
\hline
\end{tabular}

Values are presented as number (\%).

CGA, comprehensive geriatric assessment; $\mathrm{OR}$, odds ratio; $\mathrm{Cl}$, confidence interval; $\mathrm{ADL}$, physical function according to activities of daily living; IADL, instrumental $\mathrm{ADL}$; MMSE, Mini-Mental State Examination; SGDS, Korean Geriatric Depression Scale; MNA, mini nutritional assessment. 
This study only examined patients who had elective surgery. In emergency operations, elderly patients have a much greater chance of suffering from postoperative complications than younger patients. In particular, elderly patients who underwent emergency operations were reported to have 3 to 10 times higher morbidity and mortality than those who were well-prepared and underwent elective operations [27]. No studies have yet assessed the predictive value of the CGA for postoperative outcome in elderly patients in emergency settings. However, such a study would be difficult because of the lack of sufficient time to evaluate patients' conditions by interview.

One novel finding in the present study is that the eight domains of the CGA have different clinical significance. Greater independence in daily living (high ADL score) and fewer comorbidities were predictive of an uneventful recovery after surgery for CRC. Interestingly, all patients at high risk of developing delirium had major postoperative complications, although this domain showed no statistical significance on the multivariate analysis. Some previous studies did report that preoperative cognitive dysfunction was related with postoperative complications in elderly patient $[28,29]$.

The present study had several limitations. First, 80 patients did not receive preoperative CGA during the study period. The reasons for this included patient refusal, absence of a physician responsible for the CGA, and patient unavailability during the preoperative workup at the outpatient clinic. Second, this study also had bias because it was a retrospective, single-center study. However, we performed backward substitution methods with multivariate logistic regression without over fitting to reduce statistical bias. In conclusion, the results of a preoperative CGA were predictive of major postoperative complications in elderly patients undergoing surgery for CRC. Thus, its use may be clinically beneficial for detecting "high-risk" patients so that they can be provided with more careful postoperative management following surgery.

\section{CONFLICT OF INTEREST}

No potential conflict of interest relevant to this article was reported.

\section{ACKNOWLEDGMENTS}

Dr J.H.K had full access to all the data in the study and is responsible for the integrity of the data and the accuracy of the data analysis. This study was supported by a grant from the National R\&D Program for Cancer Control, Ministry for Health and Welfare, Republic of Korea (NCC grant No. 1320370).

\section{REFERENCES}

1. Park KA. 2011 Population projections for Korea (Based on the 2006 Census) [Internet]. Daejeon (KR): Statistics Korea; 2011 [cited 2011 Jun 31]. Available from: http://kostat.go.kr/portal/eng/
pressReleases/8/8/index.board.

2. Etzioni DA, Liu JH, Maggard MA, Ko CY. The aging population and its impact on the surgery workforce. Ann Surg 2003;238:1707.

3. Cramer JD, Patel UA, Samant S, Smith SS. Postoperative complications in elderly patients undergoing head and neck surgery: opportunities for quality improvement. Otolaryngol Head Neck Surg 2016;154:518-26.

4. Fukuse T, Satoda N, Hijiya K, Fujinaga T. Importance of a comprehensive geriatric assessment in prediction of complications following thoracic surgery in elderly patients. Chest 2005;127:88691.

5. Panula J, Pihlajamäki H, Mattila VM, Jaatinen P, Vahlberg T, Aarnio $\mathrm{P}$, et al. Mortality and cause of death in hip fracture patients aged 65 or older: a population-based study. BMC Musculoskelet Disord 2011;12:105.

6. Pal SK, Katheria V, Hurria A. Evaluating the older patient with cancer: understanding frailty and the geriatric assessment. CA Cancer J Clin 2010;60:120-32.

7. Kim SW, Han HS, Jung HW, Kim KI, Hwang DW, Kang SB, et al. Multidimensional frailty score for the prediction of postoperative mortality risk. JAMA Surg 2014;149:633-40.

8. Kim KI, Park KH, Koo KH, Han HS, Kim CH. Comprehensive geriatric assessment can predict postoperative morbidity and mortality in elderly patients undergoing elective surgery. Arch Gerontol Geriatr 2013;56:507-12.

9. Jung KW, Won YJ, Kong HJ, Oh CM, Lee DH, Lee JS. Prediction of cancer incidence and mortality in Korea, 2014. Cancer Res Treat 2014;46:124-30.

10. Charlson ME, Pompei P, Ales KL, MacKenzie CR. A new method of classifying prognostic comorbidity in longitudinal studies: development and validation. J Chronic Dis 1987;40:373-83.

11. Topinková E, Mádlová P, Fialová D, Klán J. New evidence-based criteria for evaluating the appropriateness of drug regimen in seniors. Criteria STOPP (screening tool of older person's prescriptions) and START (screening tool to alert doctors to right treatment). Vnitr Lek 2008;54:1161-9.

12. Stone SP, Ali B, Auberleek I, Thompsell A, Young A. The Barthel index in clinical practice: use on a rehabilitation ward for elderly people. J R Coll Physicians Lond 1994;28:419-23.

13. Clavien PA, Barkun J, de Oliveira ML, Vauthey JN, Dindo D, Schulick RD, et al. The Clavien-Dindo classification of surgical complications: five-year experience. Ann Surg 2009;250:187-96.

14. Corti MC, Guralnik JM, Salive ME, Sorkin JD. Serum albumin level and physical disability as predictors of mortality in older persons. JAMA 1994;272:1036-42.

15. Fried LP, Kronmal RA, Newman AB, Bild DE, Mittelmark MB, Polak JF, et al. Risk factors for 5-year mortality in older adults: the Cardiovascular Health Study. JAMA 1998;279:585-92.

16. Volpato S, Leveille SG, Corti MC, Harris TB, Guralnik JM. The value of serum albumin and high-density lipoprotein cholesterol in defining mortality risk in older persons with low serum cho- 
lesterol. J Am Geriatr Soc 2001;49:1142-7.

17. Ihedioha U, Gravante G, Lloyd G, Sangal S, Sorge R, Singh B, et al. Curative colorectal resections in patients aged 80 years and older: clinical characteristics, morbidity, mortality and risk factors. Int J Colorectal Dis 2013;28:941-7.

18. Tran Ba Loc P, du Montcel ST, Duron JJ, Levard H, Suc B, Descottes B, et al. Elderly POSSUM, a dedicated score for prediction of mortality and morbidity after major colorectal surgery in older patients. Br J Surg 2010;97:396-403.

19. Savlovschi C, Serban D, Trotea T, Borcan R, Dumitrescu D. Postsurgery morbidity and mortality in colorectal cancer in elderly subjects. Chirurgia (Bucur) 2013;108:177-9.

20. Jones C, Badger SA, Epanomeratikis E, McKie LD, Diamond T, Taylor MA. Role of carcinoembryonic antigen as a marker for colorectal liver metastases. Br J Biomed Sci 2013;70:47-50.

21. Alves A, Panis Y, Mathieu P, Mantion G, Kwiatkowski F, Slim K, et al. Postoperative mortality and morbidity in French patients undergoing colorectal surgery: results of a prospective multicenter study. Arch Surg 2005;140:278-83.

22. Turrentine FE, Wang H, Simpson VB, Jones RS. Surgical risk factors, morbidity, and mortality in elderly patients. J Am Coll Surg 2006;203:865-77.

23. Leung JM, Dzankic S. Relative importance of preoperative health status versus intraoperative factors in predicting postoperative adverse outcomes in geriatric surgical patients. J Am Geriatr Soc 2001;49:1080-5.

24. Tan KY, Kawamura Y, Mizokami K, Sasaki J, Tsujinaka S, Maeda T, et al. Colorectal surgery in octogenarian patients--outcomes and predictors of morbidity. Int J Colorectal Dis 2009;24:185-9.

25. Kristjansson SR, Nesbakken A, Jordhøy MS, Skovlund E, Audisio $\mathrm{RA}$, Johannessen $\mathrm{HO}$, et al. Comprehensive geriatric assessment can predict complications in elderly patients after elective surgery for colorectal cancer: a prospective observational cohort study. Crit Rev Oncol Hematol 2010;76:208-17.

26. Sjo OH, Larsen S, Lunde OC, Nesbakken A. Short term outcome after emergency and elective surgery for colon cancer. Colorectal Dis 2009;11:733-9.

27. Hamel MB, Henderson WG, Khuri SF, Daley J. Surgical outcomes for patients aged 80 and older: morbidity and mortality from major noncardiac surgery. J Am Geriatr Soc 2005;53:424-9.

28. Robinson TN, Wu DS, Pointer LF, Dunn CL, Moss M. Preoperative cognitive dysfunction is related to adverse postoperative outcomes in the elderly. J Am Coll Surg 2012;215:12-7.

29. Aykut K, Albayrak G, Guzeloglu M, Baysak A, Hazan E. Preoperative mild cognitive dysfunction predicts pulmonary complications after coronary artery bypass graft surgery. J Cardiothorac Vasc Anesth 2013;27:1267-70. 